\title{
Computernetze
}

Helga Dickel

\section{Computernetze von Frauen für Frauen}

Wie schon Anfang der 80er Jahre gehen die Meinungen (in) der Frauen(szene) zu den Neuen Technologien auch jetzt wieder weit auseinander. Neu ist der Computer an sich nicht mehr, und er wird inzwischen als Arbeitsmittel genutzt und geschätzt. Neu ist Ende der $90 \mathrm{er}$ Jahre die Technologie bzw. die Nutzung der Datenfernübertragung in Form des Internet bzw. der Mailboxnetze. Offen sind immer noch viele Fragen, theoretische und praktische. Frauen, die derzeit zu diesem Thema die Informations- und Schulungsarbeit leisten, betonen immer wieder, daß es ganz wichtig ist, den Nutzen der verschiedenen $\mathrm{Da}$ tenfernübertragungswege für die frauenpolitische und/oder berufliche Arbeit der Frauen deutlich zu machen.

Im folgenden werden einige der in erster Linie deutschsprachigen Netze und Informationsquellen in den Computernetzen vorgestellt, um einen Eindruck über den derzeitigen Stand zu vermitteln.

\section{Frauen-Mailboxen/Women's Bulletin Board Systems}

Mailboxen bieten jeder Nutzerin eine eigene E-Mail-Adresse und damit die Möglichkeiten 1. zur persönlichen Kommunikation und 2. die Teilnahme an öffentlichen Diskussionsforen in Form von Brettern in den Boxen selbst und von Mailinglisten (s. u.). Die per E-Mail ausgetauschten/verbreiteten Informationen werden von Rechner (=Mailbox) zu Rechner (=Mailbox) per Telefonleitung mit Hilfe von Modem oder ISDNtauglichem Gerät (ISDN-Karte, Terminaladapter o.a.) übertragen. Jede einzelne Mailbox tauscht die Informationen mit einer oder mehreren anderen Mailboxen aus. Der Austausch der EMail mit einem Internet-Rechner ermöglicht E-Mail weltweit. Frauen in Deutschland haben die Wahl zwischen zwei Frauen-Mailboxnetzen, wobei sich das inhaltliche Angebot nicht grundlegend unterscheidet. Die jeweils netzeigenen Bretter tauschen die beiden Netze untereinander aus. Darüber hinaus werden Bretter aus anderen Mailboxnetzen angeboten. Die thematisch angelegten Bretter bieten die Möglichkeit, per E-Mail eigene Informationen öffentlich zu machen, Informationen anderer Nutzerinnen abzurufen und öffentlich zu kommunizieren. Das Netz selbst bietet jeweils so viel oder wenig, 
wie die beteiligten Frauen einbringen. Je mehr sich »einklinken «, desto vielfältiger und umfangreicher kann das Spektrum an Information und Kommunikation sein. Jede Teilnehmerin hat die Chance, Inhalte mitzugestalten. Bretter der Frauen-Mailboxnetze sind nur für Nutzerinnen einer Frauen-Mailbox lesund beschreibbar. Sie sind nicht über einen Internet-Zugang zu bekommen. Ein Mailbox-Zugang bietet keinen vollen Internet-Zugang.

FemNet e.V. ${ }^{1}$ Frauen erobem Mailboxnetze unterstützt seit 1994 den Aufbau eines fraueneigenen und ausschließlich Frauen zugänglichen unabhängigen Mailboxnetzes im gesamten Bundesgebiet und inzwischen auch darüber hinaus. Mailboxen gibt es in Frankfurt/M., Wiesbaden, Köln, Berlin, Heidelberg, Halle, München und Wien. Ziel ist es, den Zugang für Frauen in möglichst vielen Städten zu schaffen und auch öffentlich zu machen. Öffentliche Terminals sind derzeit zugänglich im Frauenbuchladen Köln, im Mädchentreff Wiesbaden, im Frauenzentrum Mainz, in der Weiberwirtschaft in Halle und im FrauenComputerZentrum in Berlin. Inhaltliche Struktur:

- stadtspezifische Bretter je nach Box

- über 80 FemNet-Bretter (wie z.B. /FEM/AKTUELLES/BUNDES WEIT oder /FEM/FRAUENFOR SCHUNG/DISKUSSION)

- Bretter der weiteren Mailboxnetze: WOMAN, CL (ComLink-Netz für Friedens-, Umweltpolitik und Ökologie), Z-Netz (erstes deutschsprachiges Mailboxnetz benannt nach der Mailbox-Software Zerberus), APC (Association for Progressive Communication, internationales Netz), SOLINET (Gewerkschaftsnetz) und SCHULE

- Mailinglisten als Bretter: NURFRAUEN (s.u.), BEIJING95 (Liste rund um die Weltfrauenkonferenz und ihre Nachbereitung), EASTWEST (Liste des NEWW-Network East-West-Women), NEWWBEIJING, WILD-LIST (Liste zur Lesbenforschung von Women's International Studies Europe - WISE).

FemNet hat derzeit ca. $300-400$ Nutzerinnen. Frauenverbände und -netzwerke (z.B. die Frauencomputerschulen bundesweit, Connecta - Das Frauennetzwerk e.V., INET e.V. Internationales Netzwerk Weiterbildung) nutzen die Frauen-Mailboxen inzwischen auch als internes Kommunikationsmittel. Neben öffentlichen Brettern können sich die Netzwerke eigene Bretter als geschlossene Benutzerinnengruppe einrichten lassen.

Ein spezielles Projekt von FemNet ist die Vernetzung von Mädchen bzw. Mädchenzentren. Bisher sind vernetzt: die Mädchentreffs Wiesbaden, Frankfurt/M. und Berlin, das Mädchenzentrum Osnabrück und Bietigheim. In Wiesbaden werden Mädchen demnächst über die verschiedenen Jugendzentren ebenfalls Zugang zu den FemNet-Brettern bekommen.

Obwohl über FemNet das Internet lediglich per E-Mail genutzt werden kann, wird allen beteiligten Projekten die Möglichkeit geboten, kostenlos eine eigene World Wide Web-Seite auf einem Internet-Rechner $\mathrm{zu}$ plazieren: http: //www.zerberus.de/org/femnet (im Aufbau).

WOMAN - Woman Only Mail And News e.V. ${ }^{2}$ gibt es seit 1995 und bietet den verschiedenen autonom arbeitenden Frauenmailboxen derzeit in den Städten Düsseldorf, Köln, Frankfurt, Berlin, Heidelberg und Hamburg eine organisatorische Grundlage für die $\mathrm{Zu}$ sammenarbeit. 
Inhaltliche Struktur:

- stadtspezifische Bretter je nach Box

- über 80 WOMAN - und WOA (Woman Online Archive) - Bretter (z. B. WOMAN/WISSENSCHAFT oder WOMAN/AKTUELL/WELT WEIT)

- Bretter der weiteren Mailboxnetze: FemNet, CL, SCHULE, SOLINET, APC, HRNET (Human Rights Network), ZAMIR (Friedensnetz in den ehemaligen jugoslawischen Staaten) und eine Auswahl deutschsprachiger Newsgroups (DE) aus dem USENET

- deutschsprachige Mailinglisten (s.u.): NUR-FRAUEN, FRAUENLIEBE, FEMTEC-L, FINUT-L, FEMALE-L, FRAUENINFORM, JOURNALISTINNEN-L, FRAUENONLINE-L

- internationale Mailinglisten als Bretter derzeit: BEIJING95; EUROSAPPHO (europäische Lesben-Liste), VS-ONLINE-STRAT (Liste von Virtual Sisterhood zu technischen und frauenpolitischen Fragen bzgl. Internet) und über APC die Liste EAST-WEST, Weitere Listen können bei den Sysopinnen (System-Operatorinnen) angefragt werden.

Der Zugang zu WOMAN ist direkt über eine der Mailboxen möglich und auch über das Internet für Frauen mit eigener fester IP-Adresse (z.B. 194.123.45.6, über einen Internet Service Provider zu bekommen).

WOMAN bietet auf der eigenen WorldWideWeb-Seite Informationen zu den einzelnen Frauenmailboxen und stellt kostenlos Platz für Home-Pages von Frauenprojekten und Einzelfrauen zur Verfügung (s. u.). Ein Zukunftsprojekt von WOMAN ist die Bereitstellung von vollen Internet-Zugängen für die eigenen Userinnen.

\section{Deutschsprachige frauenspezifische Mailinglisten ${ }^{3}$ nur für Frauen}

JOURNALISTINNEN-L (seit Mai 1996) wurde initiiert vom Frauen-Mailbox-Netzwerk WOMAN e.V.: in Zusammenarbeit mit dem Journalistinnenbund e.V. Die Liste ist für alle interessierten Frauen offen und wird betreut von Sabine Stampfel (Sysopin der ADA-Mailbox in Köln und Mitfrau im Journalistinnenbund) und Susanne Broos (Mitfrau im Journalistinnenbund). Inhalte: Kontakte, Tips und Infos zum/r Internet/Recherche, Veranstaltungshinweise, inhaltliche Diskussionen über Arbeitsbedingungen, Berufsbild, Frauenbild in den Medien etc.

Infos über journalistinnenbund @ada.woman.de

Bestellung: E-Mail an listserv @ada.woman.de mit dem Text in der Betreff/Subject-Zeile: subscribe JOURNALISTINNEN-L

Nachrichten an die Liste: JOURNALISTINNEN-L@ada. woman.de

FRAUENONLINE-L (seit ca. Ende 1995) ist ein Forum zu Geschlechterund Machtverhältnisen im "Cyberspace «.

Bestellungen: E-Mail an listserv @ada.woman.de mit dem Text in der Betreff/Subject-Zeile: subscribe FRAUENONLINE-L

Nachrichten an die Liste: FRAUENONLINE-L@ada.woman.de

FEMTEC-L (seit September 1996)

Initiiert und betreut von Renee Schauecker und Barbara Kleinen (ebenfalls Initiatorin des Berliner Frauen-Internet-Projekts). Schwerpunkt der Liste: Internet, technische Neuigkeiten und Entwicklungen, Aufbau eigener Angebote, Gestaltung von Web-Seiten (HTML, Java), Vernetzung von Frauen und Frauenprojekten etc.

Bestellung: E-Mail mit dem Wunsch auf Eintrag an femtec-l-request@cstu- 
berlin.de. Die Mail wird derzeit von Barbara Kleinen dann weiterverarbeitet.

Nachrichten an die Liste: femtec1@cs.tu-berlin.de

Die Liste kann ebenfalls von WOMAN-Userinnen über das Brett /WOMAN/LIST/FEMTEC-L gelesen werden. Nachrichten an die Liste müssen dann allerdings an die obige Adresse geschickt werden und nicht an das Brett.

NUR-FRAUEN (seit Anfang 1996), initiiert von Nora E. Etukudo (Sysopin der SIRENE Frauen-Mailbox in Berlin), ist für den Austausch aller denkbaren Themen unter Frauen. Hintergrund war der Wunsch, allen Frauen, die in den verschiedenen Mailboxnetzen WOMAN, FemNet, ComLink u.a. Userinnen sind, in einer Liste Gelegenheit zu geben, sich auszutauschen.

Informationen und Bestellungen: formlose E-Mail an sysopin@sirene.woman.de. Nachrichten können nur von eingetragenen Frauen geschrieben werden an: nur-frauen@woman.de. Die Liste kann ebenfalls im Brett /WOMAN/LIST/NUR-FRAUEN innerhalb von WOMAN und im Brett FEM/MAILING/NUR-FRAUEN innerhalb von FemNet gelesen werden.

FRAUENLIEBE (seit Dez. 1995) ist eine Liste für Lesben zu Lesben-Themen. Sie ist als deutschsprachiges Pendant zur in erster Linie englischsprachigen europäischen Liste EUROSAPPHO eingerichtet worden. Die Liste läuft ebenfalls über den Rechner von Nora Etukudo in Berlin. Informationen und Bestellungen: formlose $\mathrm{E}$ Mail an sysopin@sirene.woman.de Nachrichten nur von eingetragenen Frauen an: frauenliebe@woman.de

Die Liste kann ebenfalls als Brett innerhalb von WOMAN gelesen werden: /WOMAN/LIST/FRAUENLIEBE.
FRAUEN-INFORM (seit 1993, eine der ältesten deutschsprachigen Frauenlisten) ist eine Liste für Frauen in der Informatik und wird von Leonie Dreschler-Fischer moderiert.

Bestellungen: E-Mail an frauen-in form-request@informatik.uni-hamburg .de mit dem Text: subscribe <eigene email-adresse >. Die Betreffzeile bleibt frei.

Nachrichten an: frauen-inform@in formatik.uni-hamburg.de.

Informationen im WWW unter http: //kogs-www.informatik.uni-hamburg.de 1 dreschle/finform.html. Ebenfalls innerhalb von WOMAN:/WOMAN /LIST /FRAUEN-INFORM

FEMALE-L (seit Dez. 1994) ist die Mailingliste des Projekts FEMinistische ALternativE im Internet der Interuniversitären Koordinationsstelle für Frauenforschung und Frauenstudien in Linz, Österreich. Die Liste wird moderiert und mit Informationen aus der Koordinationsstelle versorgt. Bestellungen: EMail an listserv@alijku04.edvz.unilinz.ac.at mit dem Text: subscribe FEMALE-L < vorname> <nachname >. Die Betreffzeile bleibt leer. Nachrichten an: female-1@uni-linz.ac.at. Informationen im WWW unter http://www.ifs.unilinz-ac.at/female/female.html.

Die Liste kann ebenfalls als Brett innerhalb von WOMAN gelesen werden: /WOMAN/LIST/FEMALE-L

FRAUEN-PHYSIK (seit November 1996) wurde angeregt auf dem Treffen der Physikerinnen in Göttingen. Die Liste wird moderiert. Fragen bzgl. der Liste an: cipmjd-owner@physik.uni-muen chen.de. Besteilungen: E-Mail an cipmjd@physik.uni-muenchen.de mit dem Text: subscribe frauen <eigene Email-Adresse>, Betreffzeile bleibt leer. Nachrichten an: frauen@physik.unimuenchen.de . WWW-Seite der Physikerinnen unter http://www.uni-ham burg.de / fb12-p1/physfrau.htm 
FINUT-L (seit Nov. 1996), geführt von Frauen in Naturwissenschaft und Technik, NUT e.V., ist ein Forum für alle Frauen in Naturwissenschaft und Technik. Bestellungen: formlose E-Mail an maillists@nut.woman.de. Nachrichten nur von eingetragenen Frauen an: finut1@nut.woman.de. Die Liste kann auch in WOMAN im Brett /WOMAN/NUT/ FINUT-L gelesen werden. Infos im WWW: http://tal.cs.tu-berlin.de/ babajaga/NUT/

\section{Deutschsprachige Frauen-Seiten im World Wide Web ${ }^{4}$}

Frauen-Internet-Projekt Hamburg http: //www.comlink.de/frauen/page1.html

Hamburger Frauenprojekte im Internet, Hamburger Frauenstadtplan, Termine in Hamburg, Links zu deutschen und internationalen Frauen-Seiten im WWW (sehr umfangreich in verschiedene Rubriken unterteilt), Archiv mit Artikeln zu Frauen und Internet im Volltext etc. Sehr lobenswert ist ein grafischer Seitenplan, der den Überblick sehr erleichtert. Zur Weihnachtszeit gab es einen Adventskalender des Künstlerinnenarchivs. In Planung: Einbindung der Video-Datenbank von Bildwechsel Hamburg.

Frauen-Internet-Projekt Berlin http: //wwwwbs.cs.tu-berlin.de/kultur/frauen/

Berliner Frauenprojekte, Berliner Frauenszene mit Infos, Termine, $\mathrm{Ca}$ fés/Kneipen, Kultur etc., Berliner Hochschulen, Links zu deutschen und internationalen Frauenseiten im WWW

\section{WOM@N http:/www.woman.de}

Informationen zu WOMAN und den beteiligten Frauen-Mailboxen, wom@n aktuell, Verzeichnis von deutschen Frauenprojekten sortiert nach verschiedenen Kategorien (nicht beschränkt auf woman-Userinnen), Persönliche Homepages von Frauen, Links zu weiteren
Frauenseiten, Startseite für Recherchen über Suchmaschinen im Internet.

\section{CeiberWeiber http:/www.ceiberweiber. com/home.htm}

Internetseiten des Projekts OnlineNOW, ein österreichisches Projekt der EU-Initiative New Opportunities for Women; Kurzbeschreibungen und Kontaktadressen zu den einzelnen NOWProjekten in Österreich und deren Partnerinnenprojekte.

\section{Pheminist Cyber RoadShow http:/ www.oeh. uni-linz.ac.at/-lisa/}

Eine der ersten deutschsprachigen Seiten im WWW zusammengestellt von Elisabeth Binder. Inhalt: Texte mit Erläuterungen zum Internet (einzelne Dienste, Netiquette, Suchmöglichkeiten), Ubersicht zu frauenspezifischen Mailinglisten und Newsgruppen, Links zu Texten über Frauen und Internet, über Pornographie und Zensur im Internet.

\section{Frauen an Hochschulen im WWW}

* Übersichtsseite zu Frauen an Hochschulen (mit teils leider veralteten Links): http://www.th-darmstadt.de /fsmathe/basin/Frauen.html

^ Interdisziplinäres Frauenforschungs-Zentrum, Universität Bielefeld:http://www.uni-bielefeld.de/IFF/

^ Uni-Frauen, Universität Bielefeld http://www.uni-bielefeld.de/IFF/

fraueninfonetz/index.html

* Institut für Frauenforschung, FH Kiel (erst im Aufbau) http://www.fhkiel.de/www/documents/fhfrauen forschung.ht $\mathrm{ml}$

* Freiburger Frauenstudien. Zeitschrift für interdisziplinäre Frauenstudien http://www.uni-freiburg.de /philfak3/eng/ffs.htm

^ Koordinationsstellen für Frauenforschung und Frauenstudien in Österreich:

Universität Wien http://www.uni vie.ac.at/Frauenforschung Universität Linz http://www.ifs.uni 
linz.ac.at/female/female.html Universit ät Graz http://www.kf unigraz.ac.at/kffwww/

\section{Frauenspezifische Datenbanken im $W W W$}

\section{ARIADNE - Kooperationsstelle für frauenspezifische Information und Do- kumentation}

ist eine frauenspezifische Servicestelle an der Österreichischen Nationalbibliothek (ÖNB), die für die »Ermittlung und Beschaffung der für die Frauenforschung/Feministische Forschung relevanten Literatur « zuständig ist. Der Bestand an frauenspezifischen Büchern der ÖNB kann im WWW über den Österreichischen Verbundkatalog BIBOS-OPAC (http://bibopac.univie. ac.at) recherchiert werden. Die ARIADNE-Datenbank für unselbständige Literatur (Zeitschriftenaufsätze, Beiträge in Sammelwerken, Graue Literatur etc., hauptsächlich deutsch- und englischsprachig) enthält ca. 10000 Dokumente und ist als eigenständige Datenbank im WWW, Zugang über http: //www.onb.ac.at/ariaddb.htm, angeboten. Die einzelnen Einträge sind formal und inhaltlich, teilweise mit Abstract und Kommentar, erschlossen. Als Grundlage für die Verschlagwortung (im österreichischen »Beschlagwortung«) wird der Österreichische Frauenthesaurus "ThesaurA" verwendet (Infos siehe http://www.onb.ac.at/ariad neu.htm).

Die Recherche in der Datenbank ist über zwei Wege möglich. Zum einen kann über neun verschiedene alphabetische Register gesucht werden: Namen von Personen, Körperschaften, Titelund Schlagwörter, Aufsatz- und Buchtitel, Zeitschriften und Reihen(titel), Erscheinungsort und -jahre, (30 versch.) Sachgruppen, Signaturen und ISBN. Zum anderen kann mit den Boolschen Operatoren UND bzw. ODER in den
Feldern "Name der Person «, "Schlagwörter" (bis max. 3) und "Erscheinungsort bzw. jahr « recherchiert werden. Die Schreibweisen der Suchbegriffe in den verschiedenen Registern beispielsweise müssen bestimmten Anforderungen entsprechen. Es ist daher unbedingt notwendig, die diversen Hilfsseiten zu lesen. Leider sind die Hilfstexte zu jedem Register in einer extra Datei. Das Abrufen der Seiten ist daher sehr zeitaufwendig.

ARIADNE gibt regelmäßig als Printausgabe und Online-Version im WWW einen Newsletter heraus. Nach Sachgruppen sortiert enthält er Listen der Neuzugänge (inkl. Abstract) und die regelmäßige Rubrik NetNews, die jeweils $\mathrm{zu}$ verschiedenen Schwerpunktthemen Netzadressen vorstellt. Schwerpunkt im ARIADNE-Newsletter 23, Nov. 96 war Philosophie.

ARIADNE - Ósterreichische $\mathrm{Na}$ tionalbibliothek, Josefplatz 1 (Postadresse), Heldenplatz (Zugang), A - 1015 Wien, Tel. +43-1-53410-487, Fax +43-1-53410-437

E-Mail:ariadne@grill.onb.ac.at http://www.onb.ac.at/ariadne.htm

DOKU-GRAZ. Frauendokumentations-, Forschungs- und Bildungszentrum

Der Literaturbestand des DOKU. GRAZ ist zugänglich über EMILE, das Informationssystem für Spezialbibliotheken an der Universität Graz - http: //www-ub.kfunigraz.ac.at/Emile/ emile.html

DOKU-GRAZ. Frauendokumentations-, Forschungs- und Bildungszentrum, Elisabethstr. 32, A - $8010 \mathrm{Graz}$

\section{KVINFO-Center for Information om Kvinde- og konsforskning}

Der Name KVINFO setzt sich zusammen aus den dänischen Begriffen KVINder $=$ Frauen, INFOrmation und FOrskning $=$ Forschung. Das dänische Informationszentrum für Frauen- und Geschlechterforschung, Mitte der 60er 
Jahre in der Königlichen Bibliothek in Kopenhagen begonnen, wurde während der 80er Jahre mit einem festen Etat des Kultusministeriums ausgestattet. Seit 1987 wird der internationale Literaturbestand ( $40 \%$ dänisch, $60 \%$ international, hauptsächlich englischsprachig) in der von verschiedenen dänischen $\mathrm{Bi}$ bliotheken geführten Datenbank DANBIB erfaßt. Der Datenbestand (Bücher und Aufsätze) ist heute ebenfalls Teil der Datenbank der Königlichen Bibliothek REX und damit im WWW recherchierbar.

Über die Homepage von KVINFO

http://www.kulturnet.dk/homes/

kvinfo/kvinfoe.htm (englischsprachige Homepage) auf den Link »Research Library«. Dort gibt es weitere Anweisungen und einen Link $\mathrm{zu}$ "REX1 «, dem Teil von REX, in dem die Datenbank von KVINFO enthalten ist.

Wenn dieser Link nicht funktioniert, Zugang über http://www.kb.dk/kb /rex/, den direkten Zugriff über die Königliche Bibliothek. Hier wiederum REX1 wählen. Über den Link "Change Database« die Datenbank von KVINFO auswählen und recherchieren. Die englische Hilfsseite ist leider noch nicht aufgebaut (Stand: Ende Nov. 96). Es gibt die Möglichkeit, über verschiedene Indices zu suchen, die Boolschen Operatoren zu benutzen, zwischen einfacher bzw. raffinierter Suchmaske oder dem Expertinnenmodus auszuwählen.

TIP: recherchiert über Titel eure Suchbegriffe, laßt euch einzelne Treffer im ausführlichen Format anzeigen und schreibt euch die dänischen Schlagworte, mit denen die Titel verschlagwortet sind, heraus. Anschließend könnt ihr mit diesen Begriffen gezielter weiterrecherchieren.

KVINFO, Nyhavn 22, DK - 1051 Kopenhagen, Tel. +45-33-135088, Fax +4533-141156, E-Mail: kvingo@inet.unic.dk

http://www.kulturnet.dk/homes/ kvinfo/
IIAV - Internationaal Informatiecentrum en Archief voor de Vrouwenbeweging

gegründet1935, ist das nationale Informations- und Dokumentationszentrum für Frauen, Frauenbewegung und Frauenforschung der Niederlande. Die Sammlung an Büchern, Zeitschriften, thematischen Dossiers, Presseartikeln, Nachlässen, Fotografien, Plakaten etc. umfassen einen Zeitraum von mehreren Jahrhunderten. Das älteste Buch ist 1578 erschienen. Auf Anfrage werden Literaturlisten und Kopien gegen Kostenerstattung verschickt.

Die Datenbank mit über 80000 Dokumenten ist in die zwei Bereiche Kata$\log$ und Archivmaterial aufgeteilt und ist zugänglich über http://www.iiav.nl /wwwopac/index-gb.html (englischsprachig). Die Datenbank »Catalogue« enthält die recherchierbaren Felder »Titel«, "Serientitel«, "AutorInnen«, "Thesaurus-Begriff« bzw. "Schlagwort «, "Abstract « und »HerausgeberIn«. In der Archiv-Datenbank sind es die Felder "Name«, "Thesaurus-Begriff «, "zeitliche Periode« und »Abstract«. Grundlage für die Verschlagwortung der Titel ist der niederländische Frauenthesaurus. Als Tip für die Recherche gilt hier ebenfalls der bei KVINFO schon formulierte.

Das IIAV gibt verschiedene Zeitschriften und Newsletter heraus. Speziell von LOVER-Magazine on Feminism, Culture and Science werden die Abstracts der jeweils zwei zuletzt erschienenen Hefte im WWW dokumentiert.

IIAV, Obiplein 4, NL - 1094 RB Amsterdam, Tel. +31-20-6650820, Fax +3120-6654267,E-Mail:info@iiav.nl 
Internationale Übersichtsseiten zu frauenspezifischen Internet-Ressourcen

$\star$ Feminism and Women's Resources http://zeno.ibd.nrc.ca/ mansfield/fe minism

\Women's Studies Resources, InforM System University of Maryland http://www.inform.umd.edu:8080/Ed Res/Topic/WomensStudies/

$\star$ Directory of Gender Related Internet Resources for Academic Research by Helen Fallon http. //www.dcu.ie/staff/hfallon/thesis.htm

\section{Anmerkungen}

1 FemNet e.V., Koselstr. 7, 60318 Frankfurt/M., Tel. 0611-375096, Fax 0611-375097 fem-b: Berlin, Mailbox (130-6183972, Fon 030-6184095

fem-f: Frankfurt/M., Mailbox 069-553507, Fon ()69.5972752

fem-hal: Halle, Mailbox 0345-2024331, Fon 0345-2021765

fem-hd: Heidelberg, Mailbox (06221-182691. Fon 06221-182708

fem-k: Köln, Mailbox (221-5101530, Fon 0221-5509449

fem-m: Munchen, Mailbox 089-31232272, Fon 089-3149202

fem-wi: Wiesbaden, Mailbox (1611-376142. Fon 0611-375096

fem-wien: Wien, Mailbox (0)43-1-4070282, Fon (0)43-1-4070283 bzw. innerhalb von Österreich mit der entsprechenden Wiener Vorwahl.
2 WOMAN e.V. Kontakt: woman.info@woman.de

ADA Köln, Mailbox 0221-766353, Fon (0221-4202406

CONNECTA Düsseldorf, Mailbox (21131912469, Fon/Fax (021(1)4-52(1)28

FEMAIL Frankfurt, Mailbox (169-747952,

Fon (169-741-141)4

FENESTRA Hamburg, Mailbox (1404108410, Fon (140)-448901

ELEKTRA Heidelberg, Mailbox (16221801742, Fon (66221-804671

SIRENE Berlin, Mailbox 030-8932911, Fon (030)-89093758

Angeschlossene Boxen mit Frauen in der Systembetreuung: BIONIC Bielefeld Fon: 0521-175254, PRIVACY1 Bremerhaven Fon: 04755-91124, NADESHDA Düsseldorf Fon: 0211-317571, LINK-GOE Göttingen: 0551-704345.

3 Eine Liste mit frauenspezifischen internationalen bzw. europäischen Mailinglisten kann unter der Adresse

http://www.inform.umd.edu:8080/EdRes/

Topic/WomensS tudies/Computing/WMST-

Uother-lists abgerufen werden. Die Liste wird von Joan Korenman fortlaufend gepflegt.

4 Es handelt sich hier um eine Auswah! von nicht-kommerziellen Seiten mit weiterführenden Links. Inzwischen werden zunehmend von z. B. Werbeagenturen WWW-Sites für Frauen angeboten. Meist können Non-Profit-Organisationen kostenlos eine Seite dort einstellen und dienen damit als Lockmittel für (Frauen)Unternehmen, die dort Werbung und eigene WWW-Seiten bezahlt schalten. 